\title{
The Non-Relativistic Limit for the e-MHD Equations
}

\author{
Hongli Wang' and Jie Zhao ${ }^{2}$ \\ ${ }^{1}$ College of Mathematics and Information Science, North China University of Water Resources and Electric Power, \\ Zhengzhou 450011, China \\ ${ }^{2}$ Zhengzhou Huimin Middle School, Zhengzhou 450000, China
}

Correspondence should be addressed to Hongli Wang; wanghongli@ncwu.edu.cn

Received 6 August 2013; Accepted 7 November 2013; Published 30 January 2014

Academic Editors: M. M. Cavalcanti and F. Peeters

Copyright (C) $2014 \mathrm{H}$. Wang and J. Zhao. This is an open access article distributed under the Creative Commons Attribution License, which permits unrestricted use, distribution, and reproduction in any medium, provided the original work is properly cited.

We investigate the non-relativistic limit for the e-MHD equations in a three-dimension unit periodic torus. With the prepared initial data, our result shows that the small parameter problems have unique solutions existing in the finite time interval where the corresponding limit problems (incompressible Euler equations) have smooth solutions. Moreover, the formal limit is rigorously justified.

\section{Introduction}

Let us consider the following so-called electron-magnetohydrodynamics (e-MHD) system [1,2]:

$$
\begin{gathered}
\partial_{t} u+(u \cdot \nabla) u+E+\gamma u \times B=0, \\
\partial_{t} B+\frac{1}{\gamma} \nabla \times E=0, \\
u=-\frac{1}{\gamma} \nabla \times B, \quad \operatorname{div} B=0,
\end{gathered}
$$

for $(x, t) \in \Omega \times[0, T]$, where $\Omega=(\mathbb{R} / 2 \pi)^{3}$ is the 3 -dimensional torus. The unknowns are $u \in \mathbb{R}^{3}, E \in \mathbb{R}^{3}$, and $B \in \mathbb{R}^{3}$. In the above equations, the non-dimensionless parameter $\sigma$ is proportional to $1 / c$, where $c=\left(\epsilon_{0} \nu_{0}\right)^{-1 / 2}$ is the speed of light, with $\epsilon_{0}$ and $\nu_{0}$ being the vacuum permittivity and permeability. Let us notice that, as $\gamma \rightarrow 0$ in (1), we get $\nabla \times E=0\left(\Rightarrow E=\nabla p^{I}\right), \nabla \times B=0, \operatorname{div} B=0(\Rightarrow B=0)$, and the incompressible Euler equations of ideal fluid:

$$
\partial_{t} u^{I}+\left(u^{I} \cdot \nabla\right) u^{I}+\nabla p^{I}=0, \quad \operatorname{div} u^{I}=0 .
$$

The goal of this paper is to justify the above formal derivation of the incompressible Euler equations for periodic initialvalue problems (IVPs) with an emphasis on three space dimensions. Indeed, it is well known that the phenomenon of non-relativistic is important in many physical situation involving various non-equilibrium processes. For instance, important examples occur in inviscid radiation hydrodynamics [3], quantum mechanics [4], Klein-Gordon-Maxwell system [5], and so on.

The rigorous derivations of the e-MHD equation (1) from Vlasov-Maxwell system equations by a scaling limit and from Euler-Maxwell system by a quasineutral regime are obtained, respectively, in [2] and in [6]. In [7], the incompressible Euler equation (2) of ideal fluid from the e-MHD system (1) via a non-relativistic limit was gotten only in formal derivation. The aim of this work is to give a rigorous justification of the asymptotic limit using the energy method.

Let us recall that the non-relativistic limit $\gamma \rightarrow 0$ or $c \rightarrow \infty$ has been investigated in a few fields. For instance, the limit $\gamma \rightarrow 0$ has been performed in Vlasov-Poisson system in [8], in isentropic relativistic Euler equations [9], in a model system for multiple space dimensions radiation hydrodynamics in [10], and in Euler-Maxwell equations [11, 12].

Now we recall some results on the Moser-type calculus inequalities in Sobolev spaces and the local existence of smooth solutions for symmetrizable hyperbolic equations for later use in this paper.

Lemma 1 (Moser-type calculus inequalities; see $[13,14])$. Let $s \geq 1$ be an integer. Suppose $u \in H^{s}\left(\mathscr{T}^{3}\right), \nabla u \in L^{\infty}\left(\mathscr{T}^{3}\right)$, and 
$v \in H^{s-1}\left(\mathscr{T}^{3}\right) \cap L^{\infty}\left(\mathscr{T}^{3}\right)$. Then for all multi-indexes $|\alpha| \leq s$, one has $\left(\partial_{x}^{\alpha}(u v)-u \partial_{x}^{\alpha} v\right) \in L^{2}\left(\mathscr{T}^{3}\right)$ and

$$
\begin{aligned}
& \left\|\partial_{x}^{\alpha}(u v)-u \partial_{x}^{\alpha} v\right\| \\
& \quad \leq C_{s}\left(\|\nabla u\|_{0, \infty}\left\|D^{|\alpha|-1} v\right\|+\left\|D^{|\alpha|} u\right\|\|v\|_{0, \infty}\right),
\end{aligned}
$$

where

$$
\left\|D^{h} u\right\|=\sum_{|\alpha|=h}\left\|\partial_{x}^{\alpha} u\right\|, \quad \forall h \in \mathbb{N} .
$$

Moreover, if $s \geq 3$, then the embedding $H^{s-1}\left(\mathscr{T}^{3}\right) \hookrightarrow L^{\infty}\left(\mathscr{T}^{3}\right)$ is continuous and one has

$$
\begin{gathered}
\|u v\|_{s-1} \leq C_{s}\|u\|_{s-1}\|v\|_{s-1}, \\
\left\|\partial_{x}^{\alpha}(u v)-u \partial_{x}^{\alpha} v\right\| \leq C_{s}\|u\|_{s}\|v\|_{s-1} .
\end{gathered}
$$

Recalling the classical result on the existence of sufficiently regular solutions of the incompressible Euler equations (2), we have the following regularity result about $\left(u^{I}, p^{I}\right)$.

Lemma 2 (see $[15,16])$. Let $u_{0}^{I}$ satisfy $u_{0}^{I} \in C^{\infty}$ and $\operatorname{div} u_{0}^{I}=$ 0 . Then there exist $0<T_{*}<\infty$, the maximal existence time, and a unique smooth solution $\left(u^{I}, p^{I}\right)$ of the incompressible Euler equations (2) on $\left[0, T_{*}\right.$ ) with initial datum $u_{0}^{I}$ satisfying, for any $T_{0}<T_{*}$,

$$
\left(u^{I}, p^{I}\right) \in C^{\infty}\left(\Omega \times\left[0, T_{0}\right)\right) .
$$

This paper is organized as follows. In Section 2 we give some analytical backgrounds and the main result. The proof of the main result is given in Section 3.

\section{Main Result}

First we will state the existence of smooth local solutions for system (1) for the smooth initial data given by

$$
\left.u\right|_{t=0}=u_{0},\left.\quad B\right|_{t=0}=B_{0} .
$$

Proposition 3 (see $[2,17])$. Assume that $\left(u_{0}, B_{0}\right)$ belongs to $C^{\infty}(\Omega)$ and satisfies

$$
-\nabla \times B_{0}=\gamma u_{0}, \quad \operatorname{div} B_{0}=0 .
$$

Then there exist $0<T^{\gamma}<+\infty$, the maximal existence time, which depends only on the initial data, and a unique smooth solution $(u, B, E) \in C^{\infty}\left(\Omega \times\left[0, T^{\gamma}\right)\right)$ of the incompressible $e$ MHD equations (1) defined on $\left[0, T^{\gamma}\right)$.

For the convergence of the e-MHD system (1), our main result is stated as follows.

Theorem 4. Let $s_{0} \in \mathbb{N}$ with $s_{0}>(3 / 2)+1$. Let $u_{0}^{I} \in C^{\infty}(\Omega)$ satisfy $\operatorname{div} u_{0}^{I}=0$ and let $\left(u_{0}, E_{0}, B_{0}\right) \in C^{\infty}(\Omega)$ satisfy (8). Assume that

$$
\left\|\left(u_{0}-u_{0}^{I}, B_{0}\right)\right\|_{H^{s_{0}(\Omega)}} \leq C \gamma
$$

for some positive constant $C$ independent of $\gamma$. Let $T_{\star}$ be the maximal existence time of the smooth solution $\left(u^{I}, p^{I}\right) \in$ $C^{\infty}\left(\Omega \times\left[0, T_{\star}\right)\right)$ of the incompressible Euler equations (2). Then, for any $T_{0}<T_{\star}$, there exist constants $\gamma_{0}\left(T_{0}\right)>0$ and $C\left(T_{0}\right)>0$, depending only on $T_{0}$ and the initial data, such that the e-MHD system (1) has a classical smooth solution $(u, E, B)$, defined on $\left[0, T_{0}\right]$, satisfying

$$
\left\|\left(u-u^{I}, B-\gamma b^{I}\right)\right\|_{H^{s_{0}(\Omega)}} \leq C \gamma
$$

for all $0<\gamma \leq \gamma_{0}$ and $0<t \leq T_{0}$, where the function $b^{I}$ satisfies $-\nabla \times b^{I}=u^{I}$ and $\operatorname{div} b^{I}=0$.

\section{Proof of Theorem 4}

Now we begin to justify the convergence of e-MHD equations to incompressible Euler equations when $\gamma \rightarrow 0$. To this end, by the local existence theory and extension method, it suffices to obtain the uniform estimates of the smooth solutions to (1) with respect to the parameter $\gamma$ so as to guarantee $T^{\gamma}>T_{\star}$ for any given $T_{0}<T_{\star}$. Denote by $T=\min \left\{T_{\star}, T^{\gamma}\right\}$ and by $C>0$ a constant which depends on $T_{0}$.

3.1. Derivation of Error Equations and $L^{2}$ Estimate. Let ( $u$, $E, B)$ be the unknown solution to the problem (1) and let $\left(u^{I}, p^{I}\right)$ be the solution to the incompressible Euler equations (2) defined on $\left[0, T_{\star}\right)$ given by Proposition 3 . Denote this by

$$
(U, F, G)=\left(u-u^{0}, E-\nabla p^{I}, B-\gamma b^{I}\right),
$$

which satisfies the following problem:

$$
\begin{gathered}
\partial_{t} U+\left(U+u^{I}\right) \cdot \nabla U=-F-U \times\left(G+\gamma b^{I}\right) \\
-u^{I} \times\left(G+\gamma b^{I}\right)-U \cdot \nabla u^{I}, \\
\partial_{t} G-\frac{1}{\gamma} \nabla \times F=-\gamma \partial_{t} b^{I}, \\
U=\frac{1}{\gamma} \nabla \times G, \quad \operatorname{div} G=0
\end{gathered}
$$

with the initial data

$$
\left.(U, G)\right|_{t=0}=\left(u_{0}-u_{0}^{I}, B_{0}-\gamma b^{I}(t=0)\right) .
$$

Based on $L^{2}$-conservation of solutions to the e-MHD system, we obtain $L^{2}$ estimates of the error function $(U, F, G)$. Our basic idea is to cancel the oscillations of the electric field $F$ and the magnetic field $G$ by using the special structures of the e-MHD system.

Lemma 5. For all $0<t<T$ and sufficiently small $\gamma$, it holds that

$$
\begin{aligned}
\int_{\Omega}\left(|U|^{2}+|G|^{2}\right)(t) d x \leq & \int_{\Omega}\left(|U|^{2}+|G|^{2}\right)(t=0) d x \\
& +C \int_{0}^{t}\left(\|U\|^{2}+\|G\|^{2}\right)(s) d s \\
& +C \gamma^{2} .
\end{aligned}
$$


Proof. Taking the $L^{2}$ inner product of the first equation in the error system (12) for $U$, by integration by parts, we get

$$
\begin{aligned}
\frac{1}{2} \frac{d}{d t}(U, U) & =-(F, U)-u^{I} \times\left(G+\gamma b^{I}, U\right)-\left(U \cdot \nabla u^{I}, U\right) \\
& =A_{1}+A_{2}+A_{3}
\end{aligned}
$$

where $(\cdot, \cdot)$ stands for the $L^{2}$ inner product of two scalar or vector functions in $\Omega$. Now we estimate each term on the right-hand side of (15).

For $A_{1}$, by using the equation $U=(1 / \gamma) \nabla \times G$ in (12) and vector analysis formula

$$
\operatorname{div}(f \times g)=\nabla \times f \cdot g-\nabla \times g \cdot f
$$

we have

$$
A_{1}=-\frac{1}{\gamma}(F, \nabla \times G)=-\frac{1}{\gamma}(\nabla \times F, G) .
$$

For $A_{2}$ and $A_{3}$, using the property of the approximate solution $u^{I}$, Cauchy-Schwarz's inequality, and Minkowski's inequality, we have

$$
\begin{gathered}
A_{2} \leq C(\gamma+\|G\|)\|U\| \leq C \gamma^{2}+C\left(\|G\|^{2}+\|U\|^{2}\right), \\
A_{3} \leq C\|U\|^{2} .
\end{gathered}
$$

Combining (17) with (18), we have

$$
\frac{1}{2} \frac{d}{d t}(U, U) \leq-\frac{1}{\gamma}(\nabla \times F, G)+C\left(\|U\|^{2}+\|G\|^{2}\right)+C \gamma^{2} .
$$

Multiplying the second equation in the error system (12) by $G$, Cauchy-Schwarz's inequality, we get

$$
\frac{1}{2} \frac{d}{d t}(G, G) \leq \frac{1}{\gamma}(\nabla \times F, G)+C\|G\|^{2}+C \gamma^{2} .
$$

It follows from (19) and (20) that

$$
\frac{d}{d t} \int_{\Omega}\left(|U|^{2}+|G|^{2}\right)(t) d x \leq C\left(\|U\|^{2}+\|G\|^{2}\right)+C \gamma^{2}
$$

This completes the proof of Lemma 5.

3.2. High Order Energy Estimates. We differentiate (12) with $\partial_{x}^{\alpha}$ for a multi-index $\alpha \in \mathbb{N}^{3}$ satisfying $|\alpha| \leq s_{0}$ with $s_{0}>(3 /$ 2) +1 to get that

$$
\left(U_{\alpha}, F_{\alpha}, G_{\alpha}\right)=\left(\partial_{x}^{\alpha} U, \partial_{x}^{\alpha} F, \partial_{x}^{\alpha} G\right)
$$

satisfies the following problem:

$$
\begin{gathered}
\partial_{t} U_{\alpha}+\left(U+u^{I}\right) \cdot \nabla U_{\alpha}=-F_{\alpha}+\sum_{i=1}^{4} \mathscr{H}_{1 i}, \\
\partial_{t} G_{\alpha}-\frac{1}{\gamma} \nabla \times F_{\alpha}=-\gamma \partial_{t} b_{\alpha}^{I}, \\
U_{\alpha}=\frac{1}{\gamma} \nabla \times G_{\alpha}, \quad \operatorname{div} G_{\alpha}=0,
\end{gathered}
$$

where

$$
\begin{gathered}
\mathscr{H}_{11}=\left(U+u^{I}\right) \cdot \nabla U_{\alpha}-\left[\left(U+u^{I}\right) \cdot \nabla U\right]_{\alpha}, \\
\mathscr{H}_{12}=-\left[U \times\left(G+\gamma b^{I}\right)\right]_{\alpha}, \\
\mathscr{H}_{13}=-\left[u^{I} \times\left(G+\gamma b^{I}\right)\right]_{\alpha} \\
\mathscr{H}_{14}=-\left[U \cdot \nabla u^{I}\right]_{\alpha} .
\end{gathered}
$$

Before performing the energy estimate, we set

$$
\mathscr{E}_{s_{0}}(t)=\|(U, G)\|_{H^{s_{0}}(\Omega)} .
$$

Lemma 6. For all $0<t<T$ and sufficiently small $\gamma$, it holds that

$$
\begin{aligned}
\int_{\Omega}\left(\left|U_{\alpha}\right|^{2}+\left|G_{\alpha}\right|^{2}\right)(t) d x \leq & \int_{\Omega}\left(\left|U_{\alpha}\right|^{2}+\left|G_{\alpha}\right|^{2}\right)(t=0) d x \\
& +C \int_{0}^{t}\left(1+\mathscr{E}_{s_{0}}(s)\right) \mathscr{E}_{s_{0}}^{2}(s) d s \\
& +C \gamma^{2}
\end{aligned}
$$

Proof. Taking the $L^{2}$ inner product of the first equation in (23) with $U_{\alpha}$, one gets, by using the third equation in (23) and integration by parts, that

$$
\begin{aligned}
\frac{1}{2} \frac{d}{d t}\left(U_{\alpha}, U_{\alpha}\right) & =-\frac{1}{\gamma}\left(F_{\alpha}, \nabla \times G\right)+\sum_{i=1}^{4}\left(\mathscr{H}_{1 i}, U_{\alpha}\right) \\
& =-\frac{1}{\gamma}\left(\nabla \times F_{\alpha}, G_{\alpha}\right)+\sum_{i=1}^{4}\left(\mathscr{H}_{1 i}, U_{\alpha}\right) .
\end{aligned}
$$

By using Cauchy-Schwarz's inequality, the Moser-type calculus inequalities in Lemma 1, and Sobolev's lemma, we have that

$$
\begin{gathered}
\left(\mathscr{H}_{11}, U_{\alpha}\right) \leq C\left(\left\|\nabla\left(U+u^{I}\right)\right\|_{L^{\infty}}\left\|D^{|\alpha|-1} \nabla U\right\|\right. \\
\left.+\left\|D^{|\alpha|}\left(U+u^{I}\right)\right\|\|\nabla U\|_{H^{s_{0}-1}}\right)\left\|U_{\alpha}\right\| \\
\leq C\left(\left\|\nabla\left(U+u^{I}\right)\right\|_{H^{s_{0}-1}}\left\|D^{|\alpha|-1} \nabla U\right\|\right. \\
\left.+\left\|D^{|\alpha|}\left(U+u^{I}\right)\right\|\|\nabla U\|_{L^{\infty}}\right)\left\|U_{\alpha}\right\| \\
\leq C\left(1+\|U\|_{H^{s_{0}}}\right)\|\|_{H^{s_{0}}}^{2} \\
\leq C\left(1+\mathscr{E}_{s_{0}}(t)\right) \mathscr{E}_{s_{0}}^{2}(t) \\
\left(\mathscr{H}_{12}, U_{\alpha}\right) \leq C\left(1+\mathscr{E}_{s_{0}}(t)\right) \mathscr{E}_{s_{0}}^{2}(t) \\
\left(\mathscr{H}_{13}, U_{\alpha}\right) \leq C \mathscr{E}_{s_{0}}^{2}(t)+C \gamma^{2} \\
\left(\mathscr{H}_{14}, U_{\alpha}\right) \leq C \mathscr{E}_{s_{0}}^{2}(t) .
\end{gathered}
$$


Combining (27) with (28) together, one gets

$$
\begin{aligned}
\frac{1}{2} \frac{d}{d t}\left(U_{\alpha}, U_{\alpha}\right) \leq & -\frac{1}{\gamma}\left(F_{\alpha}, \nabla \times G_{\alpha}\right)+C\left(1+\mathscr{E}_{s_{0}}(t)\right) \mathscr{E}_{s_{0}}^{2}(t) \\
& +C \gamma^{2} .
\end{aligned}
$$

Taking the $L^{2}$ inner product of the second equation in (23) with $G_{\alpha}$, one gets, by using Cauchy-Schwarz's inequality,

$$
\begin{aligned}
\frac{1}{2} \frac{d}{d t}\left(G_{\alpha}, G_{\alpha}\right) & =\frac{1}{\gamma}\left(\nabla \times F_{\alpha}, G_{\alpha}\right)-\left(\gamma \partial_{t} b_{\alpha}^{I}, G_{\alpha}\right) \\
& \leq \frac{1}{\gamma}\left(\nabla \times F_{\alpha}, G_{\alpha}\right)+C\left\|G_{\alpha}\right\|^{2}+C \gamma^{2} \\
& \leq \frac{1}{\gamma}\left(\nabla \times F_{\alpha}, G_{\alpha}\right)+C \mathscr{E}_{s_{0}}^{2}(t)+C \gamma^{2} .
\end{aligned}
$$

Combining (29) and (30), one gets

$$
\begin{aligned}
\frac{d}{d t} \int_{\Omega}\left(\left|U_{\alpha}\right|^{2}+\left|G_{\alpha}\right|^{2}\right)(t) d x \leq & C\left(1+\mathscr{E}_{s_{0}}(t)\right) \mathscr{E}_{s_{0}}^{2}(t) \\
& +C \gamma^{2}
\end{aligned}
$$

which yields (26).

\subsection{The End of Proof of Theorem 4. Now we let}

$$
z(t)=\mathscr{E}_{s_{0}}^{2}(t) .
$$

Then it follows from Lemmas 5 and 6 that there exists $\gamma_{0}>0$, depending only on $T_{0}$, such that, for any $0<\gamma \leq \gamma_{0}$ and any $0<t<T$,

$$
z(t) \leq C z(t=0)+C \int_{0}^{t}((1+\sqrt{z}) z)(s) d s+C \gamma^{2} .
$$

Since $z(t=0) \leq C \gamma^{2}$ for some positive constant $C$, now applying Gronwall's inequality to (33), one can conclude that there exists an $\gamma_{0}>0$ sufficiently small such that, for any $0<\gamma \leq \gamma_{0}$ and any $0<t<T$,

$$
z(t) \leq C \gamma^{2}
$$

Thus, using the a priori estimate (10), by the extension argument, we can conclude that $T^{\gamma}>T_{0}$ for any $T_{0}<T_{\star}$.

The proof of Theorem 4 is complete.

\section{Conflict of Interests}

The authors declare that there is no conflict of interests regarding the publication of this paper.

\section{Acknowledgments}

This work was supported by the United Fund for Fostering Talents of NSFC-Henan (Grant no. U1204103) and the Science and Technology Research Projects of Education Department of Henan province (Grant no. 13A110731).

\section{References}

[1] A. Kingsep, K. Chukbar, and V. Yankov, Electron Magnetohydrodynamics, vol. 16, Review of Plasma Physics, New York, NY, USA, 1990, edited by B.B. Kodomtsev.

[2] Y. Brenier, N. J. Mauser, and M. Puel, "Incompressible Euler and e-MHD as scaling limits of the Vlasov-Maxwell system," Communications in Mathematical Sciences, vol. 1, no. 3, pp. 437-447, 2003.

[3] C. Rohde and W.-A. Yong, "The nonrelativistic limit in radiation hydrodynamics: I. Weak entropy solutions for a model problem," Journal of Differential Equations, vol. 234, no. 1, pp. 91-109, 2007.

[4] L. P. Horwitz and F. C. Rotbart, "Nonrelativistic limit of relativistic quantum mechanics," Physical Review D, vol. 24, no. 8, pp. 2127-2131, 1981.

[5] N. Masmoudi and K. Nakanishi, "Nonrelativistic limit from Maxwell-Klein-Gordon and Maxwell-Dirac to PoissonSchrödinger," International Mathematics Research Notices, no. 13, pp. 697-734, 2003.

[6] Y.-J. Peng and W. Shu, "Rigorous derivation of incompressible e-mhd equations from compressible euler-maxwell equations," SIAM Journal on Mathematical Analysis, vol. 40, no. 2, pp. 540$565,2008$.

[7] S. Wang, "Asymptotic limits of compressible Euler-Maxwell system in plasma physics," Procedings in Applied Mathematics and Mechanics, vol. 7, no. 1, pp. 1041005-1041006, 2008.

[8] S. Calogero and H. Lee, "The non-relativistic limit of the Nordstrm-Vlasov system," Communications in Mathematical Sciences, vol. 2, no. 1, pp. 19-34, 2004.

[9] Y. Li and Y. Geng, "Non-relativistic global limits of entropy solutions to the isentropic relativistic Euler equations," Zeitschrift fur Angewandte Mathematik und Physik, vol. 57, no. 6, pp. 960-983, 2006.

[10] C. Rohde and W.-A. Yong, "The nonrelativistic limit in radiation hydrodynamics: I. Weak entropy solutions for a model problem," Journal of Differential Equations, vol. 234, no. 1, pp. 91-109, 2007.

[11] Y. Peng and S. Wang, "Convergence of compressible EulerMaxwell equations to compressible Euler-Poisson equations," Chinese Annals of Mathematics B, vol. 28, no. 5, pp. 583-602, 2007.

[12] J. Yang and S. Wang, "The non-relativistic limit of Euler-Maxwell equations for two-fluid plasma," Nonlinear Analysis: Theory, Methods and Applications, vol. 72, no. 3-4, pp. 1829-1840, 2010.

[13] S. Klainerman and A. Majda, "Singular limits of quasilinear hyperbolic systems with large parameters and the incompressible limit of compressible fluids," Communications on Pure and Applied Mathematics, vol. 34, no. 4, pp. 481-524, 1981.

[14] A. Majda, Compressible Fluid Flow and Systems of Conservation Laws in Several Space Variables, Springer, New York, NY, USA, 1984.

[15] F. J. McGrath, "Nonstationary plane flow of viscous and ideal fluds," Archive for Rational Mechanics and Analysis, vol. 27, pp. 229-348, 1968.

[16] T. Kato, "Nonstationary flow of viscous and ideal fluids in $R^{3}$," Journal of Functional Analysis, vol. 9, pp. 296-305, 1972.

[17] Y.-J. Peng and W. Shu, "Rigorous derivation of incompressible e-mhd equations from compressible euler-maxwell equations," SIAM Journal on Mathematical Analysis, vol. 40, no. 2, pp. 540$565,2008$. 


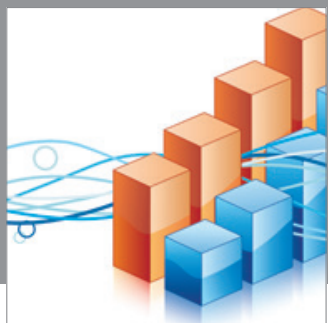

Advances in

Operations Research

mansans

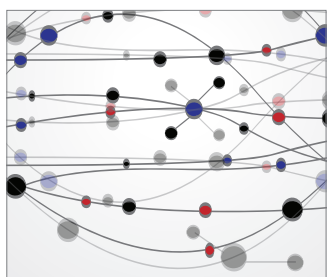

The Scientific World Journal
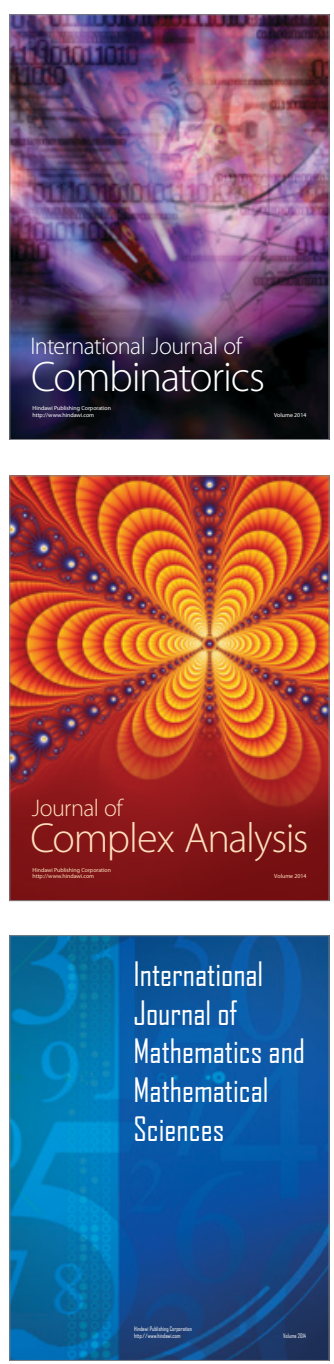
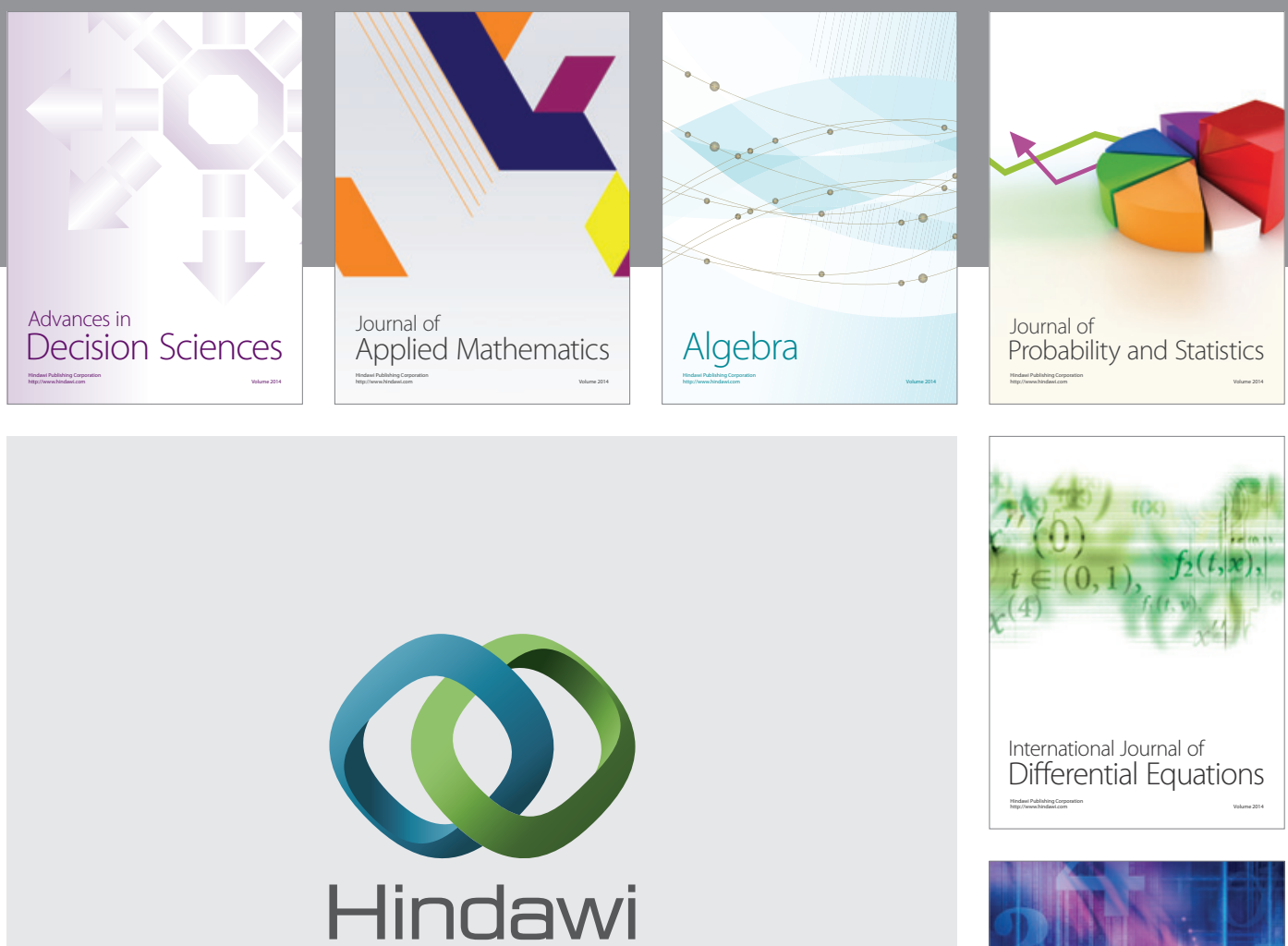

Submit your manuscripts at http://www.hindawi.com
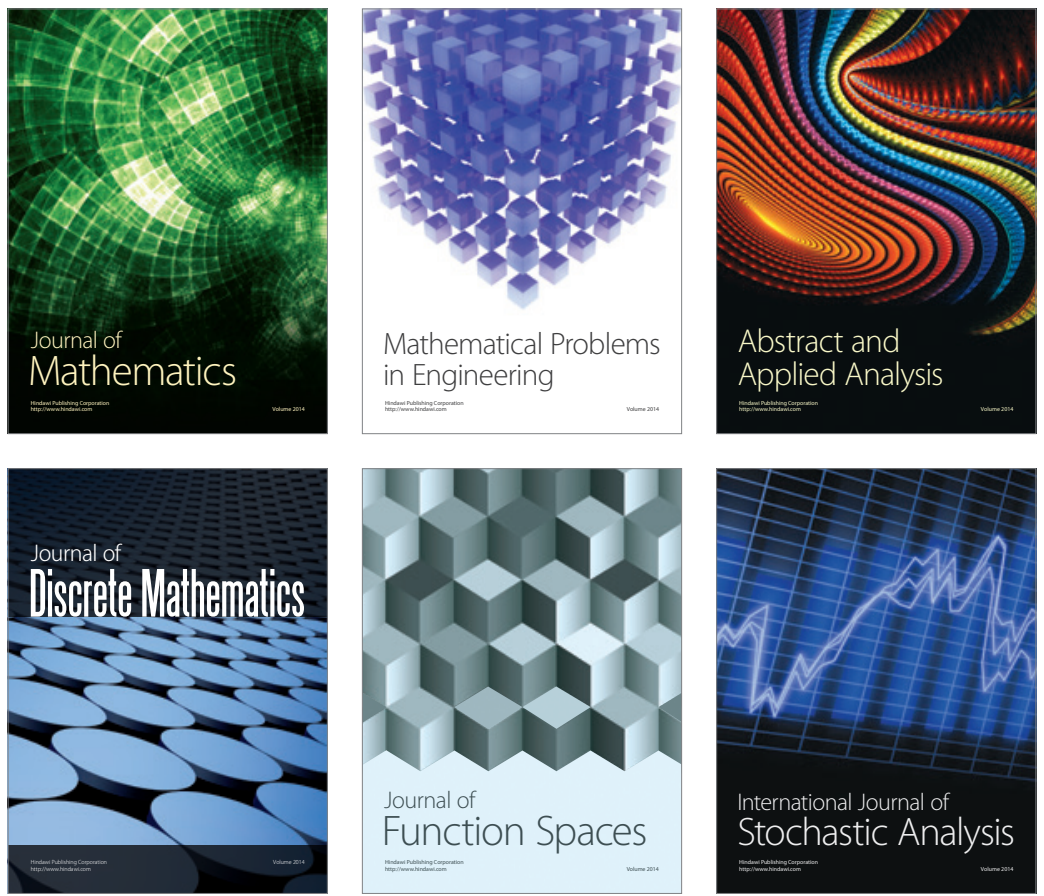

Journal of

Function Spaces

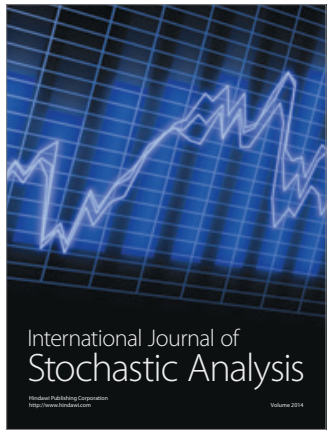

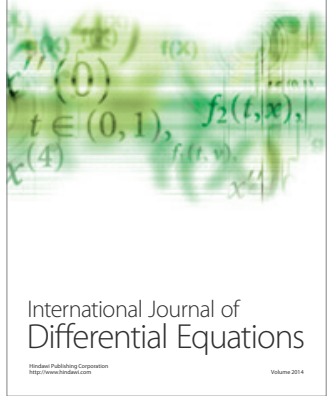
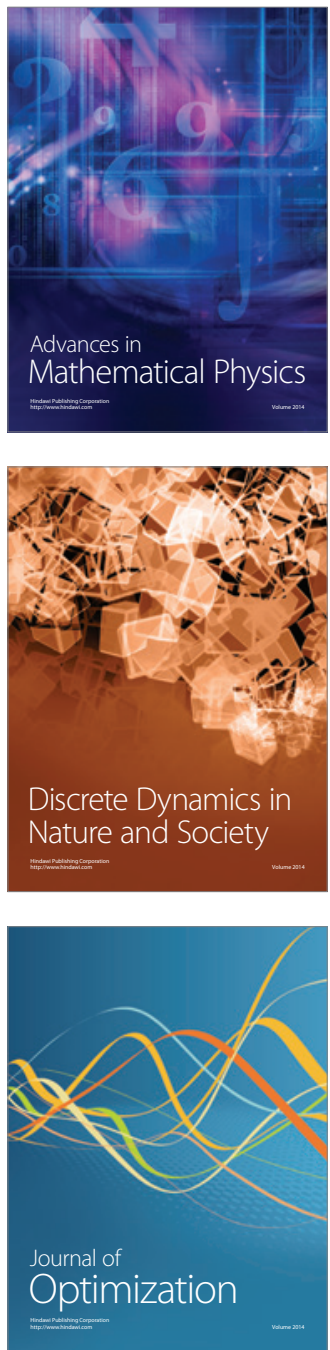